\title{
Influence of turbulent magnetic fields on mode frequencies
}

\author{
S. L. Bi ${ }^{1}$, Y. Liao ${ }^{1}$, and J. X. Wang ${ }^{2}$ \\ 1 Yunnan Astronomical Observatory and National Astronomical Observatories, Kunming 650011, PR China \\ 2 National Astronomical Observatories, Beijing 100087, PR China
}

Received 9 July 2002 / Accepted 18 September 2002

\begin{abstract}
To investigate the physical nature of solar convective zone, we employ the observed frequency shifts of solar oscillations to study the influence of magnetic perturbation inside the Sun on the low- $\ell$ solar $p$-mode oscillations. We describe the various possibility of frequency shifts for a time-dependent source of MHD turbulence. For the magnetic perturbation contribution, we obtain the frequency shifts of modes with different degree as a function of the spectrum of fluctuating magnetic field. The frequency shift is found to increase with the strength of magnetic fields in solar interior, and its temporal behavior closely follows the phase of the synthetic solar activity cycle. Our analysis indicates that the magnetic activities cause shifts of up to $0.3 \mu \mathrm{Hz}$. It is obviously shown that mode frequency, which is sensitive to the effect of magnetic fields, can be used as a diagnostic tool for the presence of turbulent magnetic fields in the convection zone.
\end{abstract}

Key words. Sun: oscillations - Sun: magnetic fields - Sun: activity

\section{Introduction}

The Sun's $p$-mode frequency is one of the most accurately known solar parameters with a precision of about $10^{-5}$ (Libbrecht et al. 1990). It is well known that the principal cause of the differences between measured and theoretical solar oscillation frequencies lies close to the solar surface, particularly where our understanding of convection is most uncertain. This information will play an important role in tying down the details of the structure of the convection.

Originally, many attempts were made to take into account the influences of purely turbulent pressure and nonadiabaticity on mode frequencies (Christensen-Dalsgaard \& Frandsen 1983; Kosovichev 1995; Gabriel 1995; Böhmer \& Rüdiger 1998; Rosenthal et al. 1999; Bi \& Xu 2000). Recently, considerable efforts have been made to study the effects of magnetic fields on the properties of the solar $p$-mode oscillations (Dziembowski \& Goode 1997; Jiménez-Reyes et al. 1998; García et al. 1999; Gavryusev \& Gavryuseva 1999; Antia et al. 2000; Moreno-Insertis \& Solanki 2000; Zhukov 2001). Up to now, more and more observational evidence collected by both space and ground-based instruments shows that the frequencies are increased by magnetic activity (Woodard \& Noyes 1985; Elsworth et al. 1994; Gelly et al. 1997; Chaplin et al. 1998; Bhatnagar et al. 1999; Howe et al. 1999; Chaplin et al. 2001; Jiménez-Reyeset et al. 2001). For low- $\ell$ solar $p$-modes, the relative change in frequency between solar activity minimum and maximum is of order $0.4 \mu \mathrm{Hz}$.

Send offprint requests to: $\mathrm{S}$. L. Bi, e-mail: wbyw@public.km.yn.cn
The sources of solar $p$-mode frequency shifts are mainly related to non-adiabatic effect, magnetic perturbation and turbulent processes. In the development of such a theory, the role is to explore details of the physical processes that dominate the mode oscillations. Our main question is whether or not the direct magnetic field fluctuation is sufficient to explain the changes in mode frequencies with the solar cycle.

In this paper, the magnetic perturbation is considered as a first approximation of the dynamic motions to the steady state. In Sect. 2, the mathematical description of the shifts is derived in detail. For the specific case, the frequency shift of solar oscillations is a fundamental consequence of the properties of magnetic perturbation. In Sect. 3, we give a simple synthetic spectrum of magnetic perturbation in a medium with zero mean magnetic field. In Sect. 4, we calculate the frequency shifts due to magnetic perturbation and compare them with helioseismic observation.

\section{Basic physical formulation}

\subsection{Mean field magnetohydrodynamics}

We consider an ideal MHD turbulence in a homogeneous, isotropic and stationary medium. If there is no rotation, the magnetohydrodynamic equations describing the motions of a fluid of total density $\rho$, pressure $P$, velocity vector $\boldsymbol{v}$, in the presence of a magnetic field $\boldsymbol{B}$, are given by (Unno et al. 1989)

$\frac{\partial \rho}{\partial t}+\nabla \cdot(\rho \boldsymbol{v})=0$

$\rho\left(\frac{\partial}{\partial t}+\boldsymbol{v} \cdot \nabla\right) \boldsymbol{v}=-\nabla P+\frac{1}{4 \pi}(\nabla \times \boldsymbol{B}) \times \boldsymbol{B}-\rho \boldsymbol{g}$ 
and the magnetic field $\boldsymbol{B}$ is described in the MHD approximation by the induction equation

$$
\frac{\partial \boldsymbol{B}}{\partial t}=\nabla \times(\boldsymbol{v} \times \boldsymbol{B}),
$$$$
\nabla \cdot \boldsymbol{B}=0
$$

where the gravitational acceleration $\boldsymbol{g}$ can be written as the gradient of gravitational potential $\boldsymbol{\Phi}$ :

$\boldsymbol{g}=\nabla \boldsymbol{\Phi}$,

and $\boldsymbol{\Phi}$ is determined by Poisson's equation

$\nabla^{2} \boldsymbol{\Phi}=-4 \pi G \rho$,

$G$ being the gravitational constant.

Based on an averaging technique similar to that developed by Stein \& Nordlund (1991), the quantities can be meaningfully divided into their mean and fluctuating parts:

$\rho=\bar{\rho}+\rho^{\prime}, \quad \boldsymbol{v}=\overline{\boldsymbol{v}}+\boldsymbol{u}, \quad P=\bar{P}+P^{\prime}, \quad \boldsymbol{B}=\overline{\boldsymbol{B}}+\boldsymbol{B}^{\prime}$,

where an overbar denotes averaged quantities, a prime the deviation from the mean, except for velocity $\boldsymbol{v}$. Note that $\boldsymbol{u}$ is turbulent velocity.

Taking into account that the fluctuating components have zero average

$\overline{\rho^{\prime}}=\overline{P^{\prime}}=\overline{\boldsymbol{B}^{\prime}}=\overline{\boldsymbol{u}}=0$,

and, if the velocity field is solenoidal

$(\overline{\boldsymbol{B}} \cdot \nabla) \overline{\boldsymbol{v}}-(\overline{\boldsymbol{v}} \cdot \nabla) \overline{\boldsymbol{B}}=0$,

after an averaging procedure, we obtain the following equations for the mean values $\left(\frac{\mathrm{d}}{\mathrm{d} t} \equiv \frac{\partial}{\partial t}+\overline{\boldsymbol{v}} \cdot \nabla\right)$

$\frac{\mathrm{d} \bar{\rho}}{\mathrm{d} t}+\nabla \cdot(\bar{\rho} \overline{\boldsymbol{v}})=0$

$\bar{\rho} \frac{\mathrm{d} \overline{\boldsymbol{v}}}{\mathrm{d} t}=-\nabla\left(\bar{P}+\bar{P}_{\mathrm{B}}\right)-\nabla \cdot(\bar{\rho} \boldsymbol{T})+\bar{\rho} \overline{\boldsymbol{g}}$

$$
+\frac{1}{4 \pi}\left[\left(\nabla \times \boldsymbol{B}^{\prime}\right) \times \overline{\boldsymbol{B}}+(\nabla \times \overline{\boldsymbol{B}}) \times \boldsymbol{B}^{\prime}\right],
$$

$\frac{\partial \overline{\boldsymbol{B}}}{\partial t}=\nabla \times(\overline{\boldsymbol{v}} \times \overline{\boldsymbol{B}}+\mathcal{E})$,

$\nabla \cdot \bar{B}=0$

where the magnetic pressure is defined as

$\bar{P}_{\mathrm{B}} \equiv \frac{\bar{B}^{2}}{8 \pi}$

and the sum of the Reynolds stress tensor $\boldsymbol{T}_{\mathrm{t}}$ and the magnetic fluctuating tensor $\boldsymbol{T}_{\mathrm{B}}$ is defined as

$\boldsymbol{T} \equiv \boldsymbol{T}_{\mathrm{t}}+\boldsymbol{T}_{\mathrm{B}}$

with

$\boldsymbol{T}_{\mathrm{t}}=\langle\boldsymbol{u} \boldsymbol{u}\rangle, \quad \boldsymbol{T}_{\mathrm{B}}=-\frac{1}{4 \pi \bar{\rho}}\left\langle\boldsymbol{B}^{\prime} \boldsymbol{B}^{\prime}\right\rangle$.

It should be noted that we assume that the variation of mean field $\overline{\boldsymbol{B}}$ along the magnetic field lines can be neglected, i.e., $\overline{\boldsymbol{B}} \cdot \nabla \overline{\boldsymbol{B}}=0$. Also, $\mathcal{E}=\left\langle\boldsymbol{u} \times \boldsymbol{B}^{\prime}\right\rangle$ is the mean electromotive force.

\subsection{Oscillation equations}

Equations (10)-(13) describing the motion of mean flow are non-linear equations. It is extremely difficult to directly solve these equations to obtain the various properties of solar oscillation. The usual method adopted is to linearize the non-linear equations. In the present paper, the main idea is that we assume a "free MHD turbulence", i.e., the MHD turbulent medium evolves freely and the MHD turbulent quantities are not perturbed by oscillations. If we consider Euler perturbations, the various physical quantities can be treated as the sum of equilibrium value and a perturbation

$\bar{\rho}=\rho_{0}+\rho^{(1)}, \quad \overline{\boldsymbol{v}}=\boldsymbol{v}_{0}+\boldsymbol{v}^{(1)}, \quad \bar{P}=P_{0}+P^{(1)}$,

where the subscript " 0 " refers to equilibrium values and the superscript "(1)" refers to perturbation values. When no motion exists in the unperturbed state, that is $\boldsymbol{v}_{0}=0$.

Substituting Eq. (16) into the nonlinear Eqs. (10) and (11) while neglecting terms with higher orders of perturbation values, and noting that the equilibrium values also fulfil these equations, we can obtain the following equations in the first order of the oscillation

$\frac{\partial \rho^{(1)}}{\partial t}+\nabla \cdot\left(\rho_{0} \boldsymbol{v}^{(1)}\right)=0$

$\rho_{0} \frac{\partial \boldsymbol{v}^{(1)}}{\partial t}=-\nabla P^{(1)}-\nabla \cdot\left(\rho^{(1)} \boldsymbol{T}\right)+\rho^{(1)} \boldsymbol{g}+\rho_{0} \boldsymbol{g}^{(1)}$

$$
+\frac{1}{4 \pi}\left[\left(\nabla \times \boldsymbol{B}^{\prime}\right) \times \overline{\boldsymbol{B}}+(\nabla \times \overline{\boldsymbol{B}}) \times \boldsymbol{B}^{\prime}\right],
$$

where the pulsating velocity is defined as $\boldsymbol{v}^{(1)} \equiv \frac{\partial \xi}{\partial t}$, and $\xi$ is the pulsating displacement vector. Obviously, we assumed that the ratio of the magnetic pressure to the thermal pressure $\left(\bar{P}_{\mathrm{B}} / P\right)$ is small because of the helioseismic constraints on solar models (Gough et al. 1996), thus the magnetic pressure term can be ignored here.

In addition, the induction equation for the fluctuating magnetic field in its linearized version is

$\frac{\partial \boldsymbol{B}^{\prime}}{\partial t}=\nabla \times\left(\boldsymbol{v}^{(1)} \times \overline{\boldsymbol{B}}\right)$.

\subsection{The effects of physics on the frequencies}

We set the time dependence of eigenfunctions $\left(\xi, P^{(1)}, \rho^{(1)} \ldots\right)$ as $\exp (-i \omega t), \omega$ being the frequency. By using the energy equation the pressure perturbation can be expressed in terms of other variables, it follows that (e.g. Christensen-Dalsgaard 1998)

$\frac{P^{(1)}}{P_{0}}=\frac{P_{\mathrm{ad}}^{(1)}}{P_{0}}+\frac{i}{\omega} \frac{\Gamma_{3}-1}{P_{0}}\left(\rho_{0} \epsilon-\nabla \cdot \boldsymbol{F}\right)^{\prime}$,

with

$P_{\mathrm{ad}}^{(1)}=P_{0} \Gamma_{1} \frac{\rho^{(1)}}{\rho_{0}}+\xi_{\mathrm{r}}\left(\frac{\mathrm{d} \ln P_{0}}{\mathrm{~d} r}-\Gamma_{1} \frac{\mathrm{d} \ln \rho_{0}}{\mathrm{~d} r}\right)$, 
where $\boldsymbol{F}$ is the sum of radiative flux and convective flux. The adiabatic exponents $\Gamma_{1}$ and $\Gamma_{3}$ are defined by

$\Gamma_{1}=\left(\frac{\partial \ln p}{\partial \ln \rho}\right)_{\mathrm{ad}}, \quad \Gamma_{3}-1=\left(\frac{\partial \ln T}{\partial \ln \rho}\right)_{\mathrm{ad}}$.

After separation of the time dependence, with the help of the continuity equation, Eq. (18) can be written as

$\omega^{2} \xi=\boldsymbol{L}_{\mathrm{ad}}(\xi)+\delta \boldsymbol{L}(\xi)$

with

$\boldsymbol{L}_{\mathrm{ad}}(\xi)=\frac{1}{\rho_{0}} \nabla P_{\mathrm{ad}}^{(1)}-\frac{\rho^{(1)}}{\rho_{0}} \boldsymbol{g}-\boldsymbol{g}^{(1)}$,

and

$\delta \boldsymbol{L}(\xi) \equiv \delta \boldsymbol{L}_{\mathrm{q}}(\xi)+\delta \boldsymbol{L}_{\mathrm{t}}(\xi)+\delta \boldsymbol{L}_{\mathrm{B}}(\xi)$,

where the force operators defined above are related to the source terms by

$$
\begin{aligned}
\delta \boldsymbol{L}_{\mathrm{q}}(\xi)= & \frac{i}{\omega \rho_{0}} \nabla\left[\left(\Gamma_{3}-1\right)\left(\rho_{0} \epsilon-\nabla \cdot \boldsymbol{F}\right)^{\prime}\right], \\
\delta \boldsymbol{L}_{\mathrm{t}}(\xi)= & \nabla \cdot\left[\rho_{0}^{-1}(\xi \cdot \nabla) \rho_{0}+\nabla \cdot \xi\right] \boldsymbol{T}_{\mathrm{t}}, \\
\delta \boldsymbol{L}_{\mathrm{B}}(\xi)= & \nabla \cdot\left[\rho_{0}^{-1}(\xi \cdot \nabla) \rho_{0}+\nabla \cdot \xi\right] \boldsymbol{T}_{\mathrm{B}} \\
& -\frac{1}{4 \pi \rho_{0}}\left[\left(\nabla \times \boldsymbol{B}^{\prime}\right) \times \overline{\boldsymbol{B}}+(\nabla \times \overline{\boldsymbol{B}}) \times \boldsymbol{B}^{\prime}\right] .
\end{aligned}
$$

Here, $\boldsymbol{L}_{\mathrm{ad}}(\xi)$ is a linear operator on $\xi . \delta \boldsymbol{L}_{\mathrm{q}}(\xi)$ is a perturbation in the operator $\boldsymbol{L}_{\mathrm{ad}}(\xi)$ caused by non-adiabaticity. The effect of Reynolds stress appears in the operator $\delta \boldsymbol{L}_{\mathrm{t}}(\xi)$. Similarly, the effect of the magnetic field appears in the operator $\delta \boldsymbol{L}_{\mathrm{B}}(\xi)$. Note that in Eq. (19), $\boldsymbol{B}^{\prime}$ is related to $\xi$.

As is well known, the change in $\omega^{2}$ caused by the perturbation to the force operator, $\delta \boldsymbol{L}$, can be obtained from the variational principal, that is

$\delta \omega^{2}=\frac{\int \xi^{*} \cdot \delta \boldsymbol{L}(\xi) \mathrm{d} V}{\int \rho_{0}|\xi|^{2} \mathrm{~d} V}$

In this work, we mainly demonstrate that the magnetic perturbation is significant. Therefore, the relative frequency change, $\delta \omega$, caused by the effect of the magnetic field that appears in the operator $\delta \boldsymbol{L}_{\mathrm{B}}$, is to first order,

$\frac{\delta \omega}{\omega}=\frac{1}{2 \omega^{2}} \frac{\int \xi^{*} \cdot \delta \boldsymbol{L}_{\mathrm{B}}(\xi) \mathrm{d} V}{\int \rho_{0}|\xi|^{2} \mathrm{~d} V}$.

For the sake of simplicity, we only consider a turbulent magnetic field $\boldsymbol{B}^{\prime}$ in a medium with zero mean magnetic field. The result is

$\frac{\delta \omega}{\omega}=\frac{1}{2 \omega^{2}} \frac{\int\left[\rho_{0}^{-1}(\xi \cdot \nabla) \rho_{0}+\nabla \cdot \xi\right] \boldsymbol{T}_{\mathrm{B}}: \nabla \xi^{*} \mathrm{~d} V}{\int \rho_{0}|\xi|^{2} \mathrm{~d} V}$.
Let $\boldsymbol{a}_{r}, \boldsymbol{a}_{\theta}$ and $\boldsymbol{a}_{\phi}$ be unit vectors in the $r, \theta$ and $\phi$ directors, then the displacement vector can be written as

$$
\begin{aligned}
\xi(\boldsymbol{r}, t)= & \sqrt{4 \pi} \mathfrak{R}\left\{\left[\widetilde{\xi}_{\mathrm{r}}(r) Y_{\ell}^{m} \boldsymbol{a}_{r}\right.\right. \\
& \left.\left.+\widetilde{\xi}_{\mathrm{h}}(r)\left(\frac{\partial Y_{\ell}^{m}}{\partial \theta} \boldsymbol{a}_{\theta}+\frac{1}{\sin \theta} \frac{\partial Y_{\ell}^{m}}{\partial \phi} \boldsymbol{a}_{\phi}\right)\right] \exp (-i \omega t)\right\},
\end{aligned}
$$

where the spherical harmonics $Y_{\ell}^{m}(\theta, \phi)$, of degree $\ell$ and order $m$, is given by

$Y_{\ell}^{m}(\theta, \phi)=(-1)^{m} c_{\ell m} P_{\ell}^{m}(\cos \theta) \exp (i m \phi)$,

with Legendre function $P_{\ell}^{m}(\cos \theta)$, and the normalization constant $c_{\ell m}$, determined by

$c_{\ell m}^{2}=\frac{(2 \ell+1)(\ell-m) !}{4 \pi(\ell+m) !}$

Assuming that $f$ is an eigenfunction of the horizontal Laplace operator,

$\nabla_{\mathrm{h}}^{2} f=-\frac{\ell(\ell+1)}{r^{2}} f$

and, writing it out in full, Eq. (32) becomes

$\frac{1}{\sin \theta} \frac{\partial}{\partial \theta}\left(\sin \theta \frac{\partial f}{\partial \theta}\right)+\frac{1}{\sin ^{2} \theta} \frac{\partial^{2} f}{\partial \phi^{2}}=-\ell(\ell+1) f$.

For low- $\ell p$-modes, we restrict ourselves to radial oscillations (Bi \& Li 1998), that is

$\nabla \xi^{*} \approx\left[\frac{\partial \widetilde{\xi}_{\mathrm{r}}^{*}(r)}{\partial r} Y_{\ell}^{m}(\theta, \phi) \exp (i \omega t)\right] \boldsymbol{a}_{r} \boldsymbol{a}_{r}$.

Consider a mode $(n, \ell)$ with eigenvector $\xi_{n \ell}$, by making use of Eqs. (30)-(34), we finally obtain the contribution of a fluctuating magnetic field to the frequency shift, $\delta \omega_{n \ell}$, as

$\frac{\delta \omega_{n \ell}}{\omega_{n \ell}}=-\frac{1}{8 \pi \omega_{n \ell}^{2} I_{n \ell}} \int_{0}^{R} \rho_{0}^{-1}\left\langle\boldsymbol{B}^{\prime} \boldsymbol{B}^{\prime}\right\rangle_{r r} \beta_{n \ell} r^{2} \mathrm{~d} r$,

where

$I_{n \ell}=\int_{0}^{R} \rho_{0} r^{2}\left[\widetilde{\xi}_{\mathrm{r}}^{2}(r)+\ell(\ell+1) \widetilde{\xi}_{\mathrm{h}}^{2}(r)\right] \mathrm{d} r$,

and

$\beta_{n \ell}=\left\{\frac{\rho_{0}^{-1}}{r^{2}} \frac{\partial}{\partial r}\left[\rho_{0} r^{2} \widetilde{\xi}_{\mathrm{r}}(r)\right]-\frac{\ell(\ell+1) \widetilde{\xi}_{\mathrm{h}}(r)}{r}\right\} \frac{\partial \widetilde{\xi}_{\mathrm{r}}^{*}(r)}{\partial r}$.

Note that the eigenvalues and eigenfunctions depend on $\omega$.

\section{The spectrum of magnetic fluctuations}

Let us assume that the pattern magnetic perturbations can be described in the same way as the case of turbulent velocities. Consider a fluctuating magnetic field $\boldsymbol{B}^{\prime}$, at two different points in space and time, e.g., at $\boldsymbol{x}$ with $\tau$ and at $\boldsymbol{x}+\boldsymbol{r}$ with $\tau+t$. The relation tensor is then defined as

$B_{i j}(\boldsymbol{r}, t) \equiv\left\langle B_{i}^{\prime}(\boldsymbol{x}, t) B_{j}^{\prime}(\boldsymbol{x}+\boldsymbol{r}, \tau+t)\right\rangle$. 
Then, the Fourier transform $B_{i j}$ is

$\phi_{i j}(\boldsymbol{k}, \omega)=\frac{1}{(2 \pi)^{4}} \int \mathrm{d}^{3} r \int \mathrm{d} \tau B_{i j}(\boldsymbol{r}, t) \mathrm{e}^{-i(\boldsymbol{k} \cdot \boldsymbol{r}-\omega t)}$,

and its inverse transform

$B_{i j}(\boldsymbol{r}, t)=\int \mathrm{d}^{3} k \int \mathrm{d} \omega \phi_{i j}(\boldsymbol{k}, \omega) \mathrm{e}^{-i(\boldsymbol{k} \cdot \boldsymbol{r}-\omega t)}$.

For a stationary, incompressible, homogeneous and isotropic MHD turbulence, a random flow $\boldsymbol{u}$ generates a fluctuating magnetic field $\boldsymbol{B}^{\prime}$ with the same probability as $-\boldsymbol{B}^{\prime}$, and it may be assumed that the correlation $\left\langle u_{i} B_{j}^{\prime}\right\rangle=0$. Therefore, the Fourier transform of the second-order correlation product of fluctuating magnetic fields has the form (Kichatinov 1991)

$\phi_{i j}(\boldsymbol{k}, \omega)=\frac{b(k, \omega)}{4 \pi k^{2}}\left(\delta_{i j}-\frac{k_{i} k_{j}}{k^{2}}\right)$.

For simplification, we may assume that for low- $\ell p$-modes $\boldsymbol{r}$ is limited to the $z$-direction $\left(r_{x}=r_{y}=0\right)$. As a result, we have

$$
\begin{aligned}
B_{r r}(r, t)= & 2 \int_{0}^{\infty} \mathrm{d} \omega \cos \omega t \\
& \int_{0}^{\infty} \mathrm{d} k b(k, \omega)\left(\frac{\sin k r}{k^{3} r^{3}}-\frac{\cos k r}{k^{2} r^{2}}\right) .
\end{aligned}
$$

The synthetic spectrum of a given fluctuating magnetic field, $b(k, \omega)$ can be formally factored into a spatial and temporal part in a simple manner:

$b(k, \omega)=b(k) \Lambda\left(\omega-\tau_{\text {corr }}\right)$,

with

$\tau_{\mathrm{corr}} \simeq \ell_{\mathrm{corr}}^{2} / v$,

where $\tau_{\text {corr }}$ is the correlation time of the turbulence, and $\ell_{\text {corr }}=$ $\alpha_{\mathrm{MLT}} H_{\mathrm{P}}$ is the corresponding correlation length of the magnetic field fluctuations, with mixing-length constant $\alpha_{\text {MLT }}$ and vertical pressure scale $H_{\mathrm{P}}$ which is the dominant local length scale in the convection zone.

Finally, making use of the above relation, Eq. (42) becomes

$B_{r r}(r, t)=\Gamma(r) \chi\left(t-\tau_{\text {corr }}\right)$,

with

$\Gamma(r) \equiv 2 \int_{0}^{\infty} b(k)\left(\frac{\sin k r}{k^{3} r^{3}}-\frac{\cos k r}{k^{2} r^{2}}\right) \mathrm{d} k$,

and

$\chi\left(t-\tau_{\text {corr }}\right) \equiv \int_{0}^{\infty} \Lambda\left(\omega-\tau_{\text {corr }}\right) \cos \omega t \mathrm{~d} \omega$.

We assume that $\left\langle u^{2}\right\rangle$ is the spectrum of the kinetic energy of MHD turbulence, and close to Kolmogorov's one

$\left\langle u^{2}\right\rangle=a \frac{u_{0}^{2}}{k_{0}}\left(\frac{k}{k_{0}}\right)^{-5 / 3}$,

where $u_{0}^{2}$ is proportional to the mean squre turbulent velocity, $k_{0} \simeq 2 \pi / H_{\mathrm{P}}$ represents the characteristic scale of energycontaining eddies. The factor $a=0.758$ is determined by the normalization condition. The spectrum is normalized by the requirement for turbulent convection that

$\int_{0}^{\infty}\left\langle u^{2}\right\rangle \mathrm{d} k=\frac{3}{2} u_{0}^{2}$

Using assumption of energy equipartition for the local spectra, that is

$\frac{\rho\langle\boldsymbol{u} \boldsymbol{u}\rangle}{2}=\frac{\left\langle\boldsymbol{B}^{\prime} \boldsymbol{B}^{\prime}\right\rangle}{8 \pi}$

Therefore, the spatial spectrum of the magnetic fluctuations follows that (Kleeorin et al. 1996)

$b(k)=\frac{8}{9} \frac{\delta B_{0}^{2}}{k_{0}}\left(\frac{k}{k_{0}}\right)^{-1}$,

where $\delta B_{0}$ is proportional to the mean strength of fluctuating magnetic field.

The simplest assumption of temporal part of magnetic spectrum, which could be suitable for connecting the variation of turbulent magnetic field with the activity cycle, is taken to be of the form

$\chi\left(t-\tau_{\text {corr }}\right)=\frac{1-\lambda \cos \left[2 \omega_{\text {cyc }}\left(t-\tau_{\text {corr }}\right)\right]}{1-\lambda}$,

where $\lambda$ is a free parameter, $\omega_{\text {cyc }}$ is the frequency of the solar magnetic cycle, i.e., $\omega_{\text {cyc }}=2 \pi / T_{\text {cyc }}, T_{\text {cyc }}=22 \mathrm{yr}$, and the time lag $\tau_{\text {corr }}$ takes into account a smoothing effect for the time spectrum.

\section{Results}

In this work, the frequency shift induced by magnetic perturbations in the solar interior is estimated in a first order approximation, neglecting physical effects which would lead to a second order correction to the frequency for each value of $m$. In principle, the frequency shift can be calculated with the help of the prescription outlined in the previous sections. Note that the choice of free parameters, i.e., $\lambda$ and $\delta B_{0}$, may strongly influence the resulting hypothetic frequency shifts.

\subsection{The temporal behavior of frequency shift}

In order to investigate the sensitivity of the $p$-mode frequencies to changes in the activity cycle, we estimated the effects of temporal changes of fluctuating magnetic fields on the frequencies for low- $\ell$ solar $p$-modes.

We note that the free parameter $\lambda$ is related to the MHD turbulent properties. In order to match the observations of temporal behavior of frequency changes, we set the free parameter $\lambda=0.60$ here.

For a given $\delta B_{0}=50 G$, the frequency shift is a function of time, which can be seen though Eq. (44). An example of the integrated frequency shift between 1.5 and $3.5 \mathrm{mHz}$, i.e., $\delta \omega_{n \ell}(t)$, obtained in this manner over a solar cycle is plotted in Fig. 1. The numerical results corresponding to $\ell=0,3$ are estimated with JCD mode parameters (Christensen-Dalsgaard 1982). It may seem surprising that the frequency shifts are sensitive to 


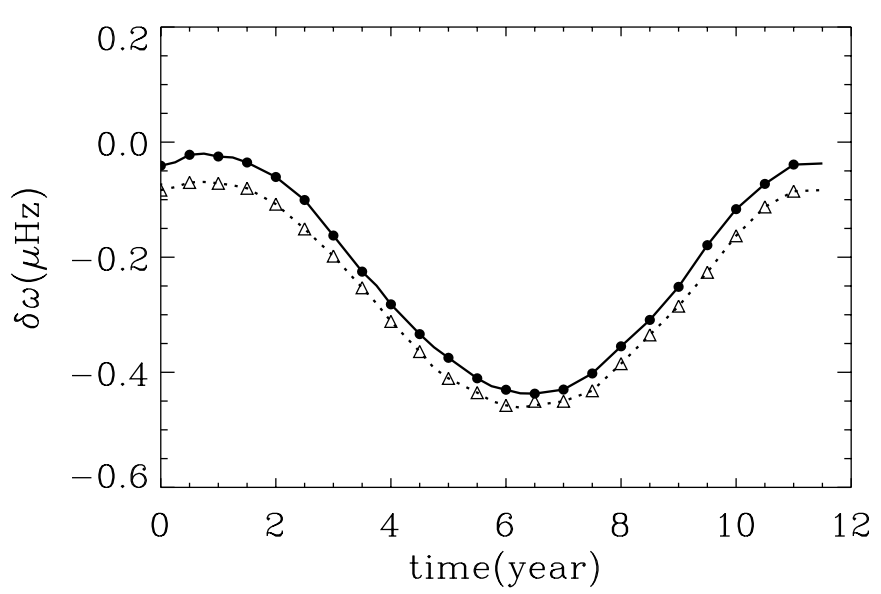

Fig. 1. Time variation of frequency shift, $\delta \omega_{n \ell}(t)$, for modes with $\ell=$ 0,3 over a complete solar cycle. The circles joined by solid line: $\ell=0$; the triangles joined dotted line: $\ell=3$.

the synthetic temporal changes of fluctuating magnetic fields, roughly consistent with the observed behavior of the solar cycle. Our result shows that the magnetic field fluctuations can cause the frequency shifts of up to about $0.3 \mu \mathrm{Hz}$. As can also be seen from Fig. 1, the modulus of the frequency shift also exhibit a slight increase with $\ell$.

Although the time variation of frequency shifts analyzed here is based on a very simple MHD turbulent model, the obtained cycle indeed shows a noticeable influence. This raises the possibility of a long-term variation of turbulent magnetic field with the activity cycle. The changes in frequency are seemingly linked to the activity cycle, but how this occurs is unclear. The calculations are not yet extensive enough to draw any conclusions about the presence of a long-term variation of turbulent magnetic field with the activity cycle, because the origin of the turbulent field itself is not well known. However, we have to convince ourselves that a frequency difference actually reflects a time variation of solar interior structure rather than any other near surface effect.

\subsection{Sensitivity of the diagnostics to the magnetic strength}

Since our analysis cannot predict the absolute value of the frequency change over the cycle because of the arbitrariness of the choice of free parameters, i.e., $\lambda$ and $\delta B_{0}$, it is interesting to check the dependence of frequency shift on the magnetic strength.

If we set $t=0$, the frequency shift mainly depends on the mean strength of the fluctuating magnetic field. As a test of this statement, we consider a case in which we artificially multiply $\delta B_{0}$ of Eq. (47). The numerical results of the frequency shifts with $\ell=0$ modes are presented in Fig. 2 for two values of the magnetic field. In Fig. 2, it is evident that the larger the magnetic field, the larger the shift of frequencies. This means that the variation of the frequency shifts reacts sensitively to the different patterns of fluctuating field corresponding to the various values of $\delta B_{0}$. As a result, Fig. 2 leads us to suspect that the spatial distribution of fluctuating fields also play an important

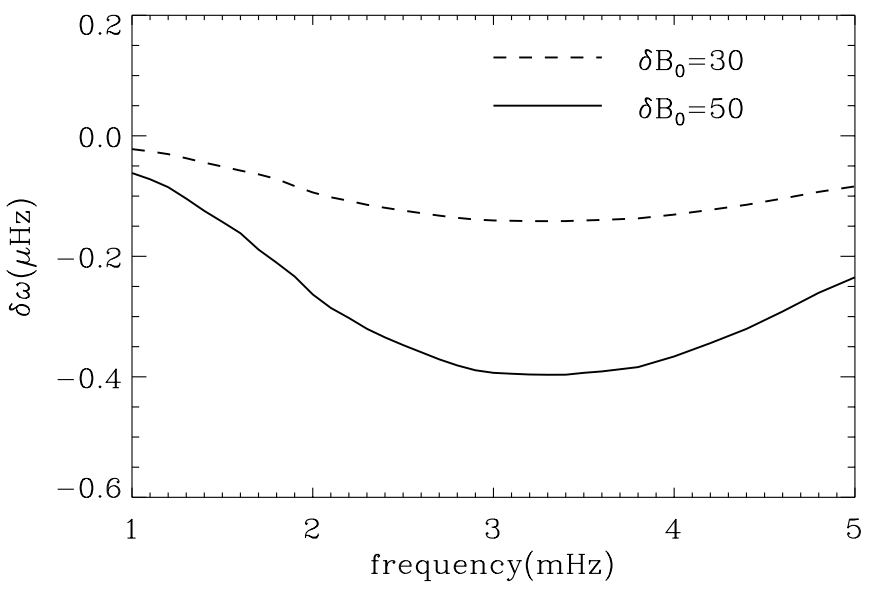

Fig. 2. Frequency shift due to the magnetic fluctuations as a function of circular frequency for the synthetic MHD turbulent spectrum. The solid and dashed lines refer to the calculations of the present formula for $\delta B_{0}=50 G$ and $\delta B_{0}=30 G$, respectively.

role in determining the shifts. It is desirable to investigate the possibility of inferring the strength of the fluctuating magnetic field in the solar interior by making use of helioseismic tool.

From the comparison between the above figures and the observational data for the frequency shifts of low- $\ell$ solar $p$-modes, we have found that magnetic perturbations are present near the bottom of the convection zone. The best fit for our calculations is obtained with strengths of $\sim 50 G$. Unfortunately, it is not sufficient to compare these shapes with the observations, since the curves of the figures closely depend on the function of the field distribution, i.e., $B_{i j}(\boldsymbol{r}, t)$. In fact, the exact pattern of $B_{i j}(\boldsymbol{r}, t)$ depends on the distribution of the fluctuating magnetic field, which is complicated by the presence of structured complexes and nonlinear processes. Furthermore, the resulting synthetic spectrum is based on our simplest model of MHD turbulence with some hypothesis and simplifications. Our results indicate that the shifts can be caused by magnetic field fluctuations, but the agreement is qualitative only.

Nevertheless, our results provide a physical explanation for the observed frequency shift of low- $\ell p$-mode oscillations, caused by the effects of the fluctuating magnetic fields near the bottom of the convection zone. Moreover, it is clear that $\delta \omega$ should be proportional to the strength of the fluctuating fields. The type of magnetic features responsible for the frequency shifts may be evidence of magnetic flux tubes or an upper limit on the strength of the fluctuating field in this region.

\section{Discussion and conclusion}

In this paper we investigate the influence of the change in strength and temporal distribution of solar turbulent magnetic fields on the frequency shifts, $\delta \omega_{n \ell}$. We have seen that the presence of a fluctuating magnetic field adds a new element to the structure of convection zone.

1. The main conclusion is that the frequency shifts of low- $\ell$ $p$-modes are sensitive to solar magnetic activities, revealing structural changes deep inside the convection zone as the solar cycle progresses. Although the results have not 
been studied in detail, they might be used as helioseismic diagnostics for the presence of turbulent magnetic fields in solar convection.

2. One possibility is that the small-scale magnetic field is created by turbulent eddies near the bottom of the convection zone. Therefore, we might hope that the consequences of such an hypothesis could be checked by helioseismology. In the long term the $p$-mode frequency changes associated with magnetic activity might provide a further calibration of a simple parameterized convection theory, such as mixing-length theory.

3. Important clues about the presence of fluctuating magnetic field near the bottom of the convection zone might provide an explanation of the magnetic flux emergence.

It must be emphasized that there are still a number of uncertainties in our analysis, foremost among which is the still open question of the type of magnetic structures responsible for the $p$-mode frequency shifts. In particular, a differential analysis of the frequency shifts with different modes can provide information on the distribution of turbulent magnetic fields. A more complicated formulation would probably have been needed in order to take into account the frequency shifts of the various $\ell$ and $m$ as the functions of the magnetic field strength and distribution.

Acknowledgements. This work was supported by NSFC through project number 10173024, and and National Key Fundamental Research Project with number G2000078401.

\section{References}

Antia, H. M., Chitre, S. M., \& Thompson, M. J. 2000, A\&A, 360, 335 Bi, S. L., \& Xu, H. Y. 2000, A\&A, 357, 300

Bi, S. L., \& Li, L. F. 1998, A\&A, 335, 673

Bhatnagar, A., Jain, K., \& Tripathy, S. C. 1999, ApJ, 521, 885
Böhmer, S., \& Rüdiger, G. 1998, A\&A, 338, 295

Chaplin, W. J., Elsworth, Y., Isaak, G. R., et al. 1998, MNRAS, 300, 1077

Chaplin, W. J., Elsworth, Y., Isaak, G. R., et al. 2001, MNRAS, 322, 22

Christensen-Dalsgaard, J. 1998, Lecture Notes in Stellar Oscillations, http://www. obs. aau.dk/jcd

Christensen-Dalsgaard, J., \& Frandsen, S. 1983, Sol. Phys., 82, 469

Christensen-Dalsgaard, J. 1982, MNRAS, 199, 735

Dziembowski, W. A., \& Goode, P. R. 1997, A\&A, 317, 919

Elsworth, Y., Howe, R., Isaak, G. R., et al. 1994, ApJ, 434, 801

Gabriel, M. 1995, A\&A, 302, 271

García, R. A., Boumier, P., Charra, J., et al. 1999, A\&A, 346, 626

Gavryusev, V. G., \& Gavryuseva, E. A. 1999, MNRAS, 303, L63

Gelly, B., Fierry-Fraillon, D., Fossat, E., et al. 1997, A\&A, 323, 235

Gough, D. O., Kosovichev, A. G., Toomre, J., et al. 1996, Science, 272, 1296

Howe, R., Komm, R., \& Hill, F. 1999, ApJ, 524, 1084

Jiménez-Reyes, S. J., Régulo, C., Pallé, P. L., \& Roca Cortés, T. 1998, A\&A, 329, 1119

Jiménez-Reyes, S. J., Régulo, C., Pallé, P. L., et al. 2001, A\&A, 379, 622

Kichatinov, L. L. 1991, A\&A, 243, 483

Kleeorin, N., Mond, M., \& Rogachevskii, I. 1996, A\&A, 307, 293

Kosovichev, A. G. 1995, Proc. of Fourth SOHO Workshop: Helioseismology (Pacific Grove, California), 165

Libbrecht, K. G., Woodard, M. F., \& Kaufman, J. M. 1990, ApJS, 74, 1129

Moreno-Insertis, F., \& Solanki, S. K. 2000, MNRAS, 313, 411

Rosenthal, C. S., Christensen-Dalsgaard, J., Nordlund, A., et al. 1999, A\&A, 351, 689

Stein, R. F., \& Nordlund, A. 1991, in Challenges to Theories of the Structure of Moderate Mass Stars, ed. D. O. Gough, \& J. Toomre (Springer, Heidelberg), 141

Unno, W., Osaki, Y., Ando, H., et al. 1989, Nonradial Oscillation (University of Tokyo Press, Tokyo)

Woodard, M. F., \& Noyes, R. W. 1985, Nature, 318, 449

Zhukov, V. I. 2001, A\&A, 369, 672 